\title{
Motivações e limitações na construção de um processo de transição agroecológica no assentamento Cachoeira no município de Itapura, São Paulo
}

\section{Motivations and limitations on construction of an agroecological transition process in the Cachoeira settlement in the municipality of Itapura, São Paulo, Brazil}

\author{
Cícero Rogério Henrique Laluce ${ }^{l}$
}

\begin{abstract}
Resumo: A agroecologia surgiu como um conjunto de saberes científicos e tradicionais que tem como objetivo fazer a transição do modelo de agricultura convencional para sistemas sustentáveis de produção. Essa nova maneira de pensar os agroecossistemas é uma forma de garantir alimentos saudáveis com inclusão social, resgate dos valores tradicionais e valorização da produção familiar e conservação do meio ambiente, no entanto, requer uma fase de transição. Assim, objetivouse analisar as principais motivações e limitações dos produtores do assentamento Cachoeira na construção do processo de transição agroecológica no município de Itapura, São Paulo. O trabalho foi uma investigação do tipo qualitativa dividida em duas etapas, na primeira foi aplicado um questionario semiestruturado para diagnóstico da realidade e na segunda etapa uma reunião com a metodologia do tipo tempestade de ideias para levantamento das motivações e limitações para a transição agroecológica no assentamento. $\mathrm{O}$ trabalho permitiu perceber que mesmo não atendendo os pré-requisitos entendidos como necessários para o processo de transição agroecológica, as famílias têm buscado construir com base na sua realidade e com seus recursos, novas estratégias para construção de um processo de mudança para alcançar autonomia, melhoria do ambiente e da qualidade de vida.
\end{abstract}

Palavras-chave: Agricultura familiar; Desenvolvimento sustentável; Comunidades rurais; Aprendizado

\begin{abstract}
Agroecology emerged as a set of scientific and traditional knowledge that aims to make the transition from the conventional agriculture model to sustainable production systems. This new way of thinking about agroecosystems is a way to guarantee healthy food with social inclusion, recovery of traditional values and valuation of family production and environmental conservation, however, it requires a phase of transition. Thus it was analyzed the main motivations and limitations of the producers of the Cachoeira settlement in the construction of the agroecological transition process in the city of Itapura, São Paulo. The study was a qualitative research divided into two stages, in the first one it was applied a semistructured questionnaire for reality diagnose and in the second stage a meeting with the methodology of the storm type of ideas to survey the motivations and limitations for the agroecological transition in the settlement. The study allowed us to understand that, despite not meeting the prerequisites understood as necessary for the agroecological transition process, families have sought to build, based on their reality and with their resources, new strategies to build a process of change to achieve autonomy, improving the environment and quality of life.
\end{abstract}

Keywords: Family Agriculture; Sustainable development; Rural communities; Learning

\footnotetext{
*Autor para correspondência

Recebido para publicação em 09/10/2017; aprovado em 19/03/2018

Mestre em Sistema de Produção; Professor da Universidade do Estado de Mato Grosso, Endereço: Av. Perimetral Deputado Rogério Silva - Norte 2, Alta

Floresta, Mato Grosso, 78580-000. E-mail: rogeriolalucci@unemat.br
} 


\section{INTRODUÇÃO}

Segundo Wezel (2009) o termo agroecologia surge inicialmente na literatura como a fusão de duas ciências, a agronomia e a ecologia, para explicar as interações entre estas ciências e outras como a sociologia, a economia e a antropologia nas inter-relações dentro dos sistemas produtivos da agricultura comercial. Nos últimos 30 anos a discussão sobre a agroecologia passa a ter duas grandes vertentes no âmbito mundial, uma norte-americana baseada em estudos voltados aos conhecimentos e manejo dos agroecossistemas e outra sob enfoque das ciências sociais e biológicas na Espanha. Segundo Moreira e Carmo (2004) estas duas vertentes têm como raízes o legado teórico de Angel Palerm e Efrain Hernandez Xolocotzi que posteriormente tiveram seus estudos refinados pelo mexicano Victor Manuel Toledo e outros cientistas norte-americanos como Stephen Gliessman e Miguel Altieri.

Segundo Caporal e Costabeber (2004) a agroecologia ainda é um conceito em construção, mas entendida como um enfoque científico destinado a apoiar a transição dos atuais modelos de desenvolvimento rural e de agricultura convencionais para estilos de desenvolvimento rural e de agriculturas sustentáveis, apoiando-se no conhecimento acumulado em diversas disciplinas científicas e nos conhecimentos das populações rurais para estudar a atividade agrária sob uma perspectiva ecológica.

$\mathrm{Na}$ prática, a mudança nos sistemas produtivos em direção à agroecologia pressupõe uma fase de transição denominada transição agroecológica. Neste processo, segundo Gliessman (2005) são necessárias três etapas: redução do uso de insumos externos; substituição de insumos químico-sintéticos por insumos orgânicos e práticas alternativas e redesenho dos sistemas produtivos, proporcionando aumento expressivo da biodiversidade. Gliessman (2001) acrescenta ainda mais uma etapa nesse processo de transição, que segundo o autor é preciso reestabelecer uma relação mais direta entre quem produz e quem consome os produtos de base ecológica, com o objetivo de construir uma cultura de sustentabilidade que leva em consideração a interação entre todos os componentes do sistema agroalimentar.

Para autores como Schmitt (2013) esta construção é um processo complexo e se desenvolve a partir de práticas concretas de manejo dos agroecossistemas em contextos socioambientais específicos, que envolvem distintas visões de mundo, e, portanto, descontinuidades e assimetrias em termos de valores, conhecimentos, interesse e poder entre os diferentes agentes envolvidos. Sendo assim, "Para além das formas de apoderar-se da natureza os agricultores, na transição agroecológica, devem absorver a ideia da coevolução entre os sistemas naturais e sociais" (CARMO, 2002.p.9)

Objetivou-se analisar as limitações e motivações das famílias do assentamento Cachoeira no munícipio de Itapura, São Paulo para a construção do processo de transição agroecológica.

\section{MATERIAL E MÉTODOS}

A metodologia utilizada foi de investigação do tipo qualitativa e os métodos para coletar as informações de interesse e alcançar os objetivos propostos da investigação foram baseados em pesquisa bibliográfica, observação de campo, aplicação de questionário e utilização de ferramenta participativa do tipo "Chuva de ideias". A chuva de ideias ou brainstorming, segundo Cimas (2009) é uma técnica que nos permite obter dados, opiniões e informações rápidas sobre um tema específico, principalmente quando se trabalha com grupos grandes e populações com baixo grau de escolaridade.

O assentamento Cachoeira está localizado nas coordenadas planas geográfica do Datum South American Datum 1969 (SAD-69) UTM 7.730.876,35 m Norte e 458.361,05 m Leste, referida ao meridiano central $51^{\circ} \mathrm{WGr}$ e Fuso 22, localizada a aproximadamente $12 \mathrm{Km}$ da cidade de Itapura/SP, seguindo pela rodovia SP 595 no sentido ItapuraIlha Solteira no Estado de São Paulo. Possui uma área total georreferenciada de 972,5791 ha dividia em 65 lotes que possuem uma área média de 13 hectares. A área de reserva legal foi constituída de forma coletiva com área de 129,0492 hectares, portanto apresentando um déficit de 65,4667 hectares em relação à legislação vigente. A principal entidade representativa dos produtores é associação dos Produtores Rurais da Cachoeirinha, fundada no ano de 2008.

O primeiro contato com a comunidade foi no mês de março de 2014, em uma reunião de apresentação formal do grupo e dos objetivos do trabalho. Posteriormente foi aplicado um questionário com perguntas abertas e fechadas em 40 lotes sorteados aleatoriamente, para diagnosticar e conhecer a realidade das famílias desta comunidade. Após os primeiros encontros e a aplicação do questionário foi realizado um segundo encontro no mês de agosto de 2014, por meio de uma reunião geral, com aproximadamente 20 famílias para divulgação dos resultados do diagnóstico realizado. Essa primeira reunião serviu para que as famílias pudessem visualizar, de forma sistematizada, sua realidade.

Em um segundo momento foram apresentadas as propostas de trabalho, elaboradas como alternativas para solucionar demandas apresentadas no primeiro diagnóstico. Estas propostas foram apresentadas em reuniões distintas, em dias alternados no mês de janeiro de 2015 e serviram, neste trabalho, como forma de motivação para que a população refletisse sobre as possibilidades de desenvolver formas mais sustentáveis de produção. As propostas foram: uso de pastoreio Voisin na produção de leite; Tecnologias viáveis para o tratamento de resíduos orgânicos; Sistema Agroflorestal para recomposição de reserva legal; Características da pecuária leiteira no assentamento Cachoeira e controle de ectoparasitas com manejo agroecológico.

Após a apresentação de cada proposta, ainda no mês de janeiro, foi realizada uma palestra por um docente da UFMSUniversidade Federal do Mato Grosso do Sul com o tema: "Agroecologia e Educação do Campo: realidade e desafios" como forma de problematização e reflexão sobre questões de educação e produção sustentável para $o$ processo de desenvolvimento do assentamento.

Por fim, utilizando a ferramenta participativa tempestades de ideias ou brainstorming, buscou-se avaliar o grau de motivação, o que já está sendo realizado e as limitações existentes para que as famílias adotem práticas mais sustentáveis no processo de desenvolvimento do assentamento.

\section{RESULTADOS E DISCUSSÃO}

Para Reichert e Gomes (2012) o agricultor que produz no sistema convencional, assistido em quase todas suas 
necessidades pelas facilidades do modelo hegemônico de produção, enfrenta problemas de diversas naturezas para viabilizar de forma econômica a unidade de produção agrícola, o agricultor familiar que está em um processo de transição enfrentando carência de informações tecnológicas sobre os processos de produção orgânica ou de base ecológica, entre outras dificuldades como falta de crédito, falta de assistência técnica apropriada, tem muito mais dificuldade nesse processo.

A tomada de decisão nas unidades de produção familiar é um processo complexo que envolve além das potencialidades econômica e ambientais, vários outros aspectos como cultura, relações sociais, preocupações com o meio ambiente, implicações nas relações familiares entre outras, ou seja, vão muito além da utilidade econômica da produção.

Mesmo assim, trabalhos têm demostrado que um grande número de produtores em diferentes regiões do Brasil e do mundo, fizeram a transição agroecológica ou estão nesse processo e que as principais motivações para o processo de transição agroecológica estão associados a fatores não econômicos como: a opinião da família, a qualidade ambiental, nível de informação (COOK, 2005); diminuição nos custos de produção, produção de alimentos livres de resíduos químicos e contaminantes, benefícios indiretos sobre o meio ambiente e a saúde (MARIN, 2009); a vida do agricultor, a saúde do consumidor e a preservação dos recursos naturais como a terra e a água (REICHERT; GOMES 2009); REICHERT et al., 2010); saúde familiar (REICHERT; GOMES 2012).

$\mathrm{Na}$ analise das principais motivações, limitações e as ações apresentadas pelas famílias na construção do processo de transição agroecológica no assentamento Cachoeira em Itapura/SP, nota-se que os resultados encontrados corroboram com os estudos anteriores, pois as principais motivações foram a busca de independência nos processos produtivos, mas principalmente, uma preocupação com a qualidade do alimento produzido e consumido (Tabela 1).

Tabela 1. Principais motivações, limitações e ações presentes na construção do processo de transição agroecológica no assentamento Cachoeira em Itapura, São Paulo

\begin{tabular}{|c|c|c|}
\hline Motivações: & Limitações & O que vem sendo feito \\
\hline $\begin{array}{l}\text { Buscar formas de produzir com pouca } \\
\text { água; }\end{array}$ & Falta de água; & Irrigação por gotejamento simples; \\
\hline $\begin{array}{l}\text { Fazer economia, ou seja, não usar } \\
\text { insumos, usar caldas e compostagem; }\end{array}$ & $\begin{array}{l}\text { Uso de agrotóxicos nas fazendas } \\
\text { vizinhas (cana) com pulverizações } \\
\text { aéreas; }\end{array}$ & $\begin{array}{l}\text { Plantio de Nim (Azadirachta indica A. } \\
\text { Juss); }\end{array}$ \\
\hline $\begin{array}{l}\text { Melhorar a saúde, pois a produção sem } \\
\text { pesticidas não contamina e não } \\
\text { intoxica }\end{array}$ & $\begin{array}{l}\text { Falta de uma ATER - Assistência } \\
\text { Técnica e Extensão Rural especifica, } \\
\text { compromissada e suficiente; }\end{array}$ & $\begin{array}{l}\text { Adubação com estercos nas hortas, } \\
\text { pomares, pasto e lavoura; }\end{array}$ \\
\hline $\begin{array}{l}\text { Fazer um resgate da autonomia, } \\
\text { principalmente das sementes; }\end{array}$ & $\begin{array}{l}\text { Pouco conhecimento das famílias } \\
\text { (erros desmotiva); }\end{array}$ & $\begin{array}{l}\text { Produção de biofertilizantes } \\
\text { (supermagro e "nitrogenados"); }\end{array}$ \\
\hline Se alimentar melhor e proporcionar & Perda da autonomia; & Cultivo mínimo; \\
\hline saúde animal, com uso de tratamentos & E medo de mudança. & Adubação verde; \\
\hline alternativos como exemplo uso de & & Compostagem. \\
\hline $\begin{array}{l}\text { preparados a base de Nim (Azadirachta } \\
\text { indica A. Juss). }\end{array}$ & & \\
\hline
\end{tabular}

As principais limitações apontadas indicam a falta de infraestrutura; ausência de um serviço de Assistência técnica e extensão rural apropriado para o processo; pressões exercidas pelos monocultivos de cana de açúcar das fazendas vizinhas; falta de conhecimento e perda de autonomia dos produtores, entendido como sua dependência de fatores externos para garantir a produção, principalmente a aquisição de sementes.

As limitações apresentadas demostram a importância da atuação do estado nos processos de desenvolvimento do campo; nesse caso criando entraves ao processo de transição para sistemas produtivos mais sustentáveis quando não viabiliza condições mínimas para que o processo se efetive como a falta de água e a falta de assistência técnica adequada. Esse fato não é só pontual, historicamente o Estado tem desempenhado papel fundamental na construção de um modelo de desenvolvimento que não privilegia os pequenos produtores, muito menos formas de desenvolvimento sustentáveis.

Ainda com relação a ATER (assistência técnica e extensão rural), ressalta-se a expressão "compromissada" apontada pelos agricultores, concordando com a observação de Caporal (2009) ao citar que:

à experiência prática está ensinando que, para desenvolver uma agricultura sustentável, os profissionais da extensão devem mudar seus compromissos e sua forma de ação, deixando de atuar como um experto transferidor de tecnologias, passando a atuar como "um facilitador que trabalha com os agricultores para aprender, desenvolver tecnologias e transformar-se em experto (CAPORAL, 2003, p.10)

Mais do que qualificada, a nova ATER deve ter um compromisso político, ético e social para que de fato a nova PNATER - Política Nacional de Assistência Técnica e Extensão Rural se efetive na prática. Ainda segundo Caporal (2009), a nova ATER deve buscar trabalhar propondo e apoiando mudanças nos sistemas produtivos atuais para processos mais sustentáveis, processos estes entendidos como resultado de novos aprendizados em novas formas de se construir conhecimento pautadas por iniciativas persistentes e destinadas a melhorar as relações dos homens entre si e destes com a natureza, respeitando os limites ecossistêmicos. Portanto, como fazer isso sem compromisso? 
A perda de autonomia e presença de fazendas vizinhas que fazem pulverizações aéreas de agrotóxicos, evidenciam uma dificuldade imposta pela territorialização do capital no território, ou seja, quando ele se apropria do território e impõe suas formas de produção, dificultando outras formas de reprodução no campo que não áquelas que favorecem seus interesses. Sua ação na busca de melhores rendimentos cria, muitas vezes, contradições internas ao próprio capital, conforme observado por Cleps (2009), ao analisar os dados do IBGE, aponta que aumentos sucessivos de área cultivada com cana de açúcar no nordeste do Estado de São Paulo e no triângulo mineiro têm diminuído o efetivo do rebanho bovino da pecuária de corte.

Pode-se analisar as limitações impostas pelo pouco conhecimento das famílias (erros desmotiva) tomando como base aquilo citado por Guzmán (2001) quando afirma que: na maior parte das zonas rurais das "sociedades avançadas" ou em regiões fortemente impactadas pela lógica da modernização, a erosão do conhecimento local foi tão forte que parece tremendamente difícil o "resgate" destes conhecimentos locais.(GUZMÁN, 2001, pg. 39)

Porém, o mesmo autor afirma que mesmo nestes ambientes é possível realizar a transição agroecológica, pois o homem possui uma capacidade intrínseca de ler os "indicadores naturais" que são oferecidos pelo ecossistema e de interpretar as inter-relações da "trama da vida". Para o autor é essa eco-lógica existente nos ciclos naturais, vinculada à cada aspecto de natureza, que possibilita a geração do conhecimento local.

Podemos discutir o medo de mudança como estratégia de sobrevivência, pois as famílias vivem sob condições adversas, pois no seu entorno predominam as relações de produção capitalistas que monopolizam e se territorializam, dificultando o acesso aos mercados, ao crédito e causando a degradação do ambiente; onde não há assistência técnica e infraestrutura adequada; onde $\mathrm{o}$ ambiente utilizado para implantação do assentamento já se encontrava degradado com solos de baixa fertilidade e onde a biodiversidade já estava comprometida. Neste cenário são poucas as possibilidades disponíveis aos camponeses. Sendo assim, sua família, sua casa, e principalmente a terra é para muitos tudo o que têm, portanto, o erro, risco inerente à mudança, pode significar perder tudo, assim podemos entender a importância das estratégias para superação das dificuldades encontradas no território, pois segundo Sant'Ana (2011)

Estas tendem a ser edificadas com base em uma tradição bastante forte e se constituem em reações, alternativas, ou adaptações referentes a alguma restrição imposta pelas condições objetivas de trabalho e de vida. (SANT'ANA, 2011, p. 291)

Mesmo diante das dificuldades apontadas as famílias vêm implementando ações na busca de um modo de vida e de produção mais sustentável, percebe-se que as famílias conhecem o caminho para o processo da mudança. A busca de práticas como as apresentadas nas principais ações das famílias representam ações concretas na tentativa de se desvencilhar das amarras da "modernização" do campo imposta pelo Capital. Assim as ações da comunidade vão de encontro ao citado por Guzmán (2001) quando cita que:

apesar da persistência deste processo de modernização, as comunidades locais geraram múltiplos mecanismos de resistência para sobreviver a um contexto fortemente hostil à natureza de suas relações, tanto das relações entre as pessoas como das pessoas com os recursos naturais. Tais formas de resistência constituem as respostas locais a uma generalizada agressão sociocultural, manifestando-se através de uma grande quantidade de elementos específicos de cada etnoecossistema (GUZMÁN, 2001.pg, 36)

Desta forma, mesmo que sem os conceitos, as famílias ao buscarem a autonomia nos sistemas produtivos, respeitando suas potencialidades e limitações, com uso eficiente dos recursos disponíveis, valorização dos saberes tradicionais e da ciência, constroem na práxis, a cada ação implementada e consolidada a sustentabilidade dentro de cada agroecossistema. Porém deve ser enfatizado que para se chegar à proposta da agroecologia não basta substituir as formas de produção convencionais por práticas mais sustentáveis, apesar destas práticas fazerem parte do processo de transição para a agroecologia.

Para Guzmán (2001) o desenvolvimento rural sustentável com enfoque agroecológico só existe se estiver baseado na agricultura e na sua articulação com o sistema sociocultural local. Para o mesmo autor isso só é possível quando se leva em conta no processo a Integralidade, ou seja, a efetivação das estratégias aplicadas ao conjunto das potencialidades e oportunidades de aproveitamento dos distintos recursos existentes na comunidade; a Harmonia e equilíbrio entre crescimento econômico e manutenção da qualidade do meio ambiente; Autonomia de gestão e controle dos elementos-chave do processo; Minimização das externalidades negativas e controle de mercados tanto na fase de produção como de comercialização; Manutenção e potencialização dos circuitos curtos de comercialização; Valorização e utilização do conhecimento local no manejo dos recursos naturais e Pluriatividade, seletividade e complementaridade da renda utilizadas como mecanismos para reforçar os laços de solidariedade.

\section{CONCLUSÕES}

As famílias do assentamento Cachoeira vêm adotando práticas alternativas para substituição de insumos químicos para conseguirem melhorar a qualidade do alimento produzido e proporcionar saúde animal; adotando práticas mais apropriados aos sistemas de produção de base ecológica como plantio direto e cultivo mínimo que melhoram o ambiente e diminuem os custos de produção, apesar das limitações de falta de infraestrutura, pressões externas do monocultivo da cana-de-açúcar, falta de assistência técnica, falta de informação sobre o processo de transição, e medo da mudança. Assim, mesmo não atendendo os pré-requisitos entendidos como necessários para o processo de transição agroecológica, as famílias têm buscado construir com base na sua realidade e com seus recursos, novas estratégias para construção de um processo de mudança para alcançar autonomia, melhoria do ambiente e da qualidade de vida.

\section{REFERÊNCIAS}

CAPORAL, F. R. Agroecologia: uma nova ciência para apoiar a transição a agriculturas mais sustentáveis. Brasília. $2009.30 \mathrm{p}$.

CAPORAL, F. R., COSTABEBER, J. A. Agroecologia e extensão rural: contribuições para a promoção do 
desenvolvimento rural sustentável. Brasília: MDA, 2004. $166 \mathrm{p}$

CARMO, M. S. Agricultura familiar e o local na transição agroecológica. Disponível em: < http://www.sober.org.br/palestra/12/090417.pdf > Acesso em: 02/04/2015

CIMAS, Observatório Internacional de Ciudadanía y Medio Ambiente Sostenible. Manual de Metodologias Participativas. Madri, 2009.

CLEPS, J. Concentração de poder no agronegócio e (Des) territorialização: os impactos da expansão recente do capital sucroalcooleiro no triangulo mineiro. Disponível em < http://www.seer.ufu.br/index.php/caminhosdegeografia/article /viewFile/16152/9091: Acesso em: 22 maio de 2017.

COCK, L. Determinants of organic farming conversion. XI International Congress of the European Association of Agricultural Economists. 2005. Disponivel em:< https://www.researchgate.net/publication/23509544_Determi nants_of_Organic_Farming_Conversion > Acesso em $10 \mathrm{de}$ maio de 2018 .

IBGE, INSTITUTO BRASILEIRO DE GEOGRAFIA E ESTATÍSTICA. Censo Agropecuário 2006. Disponível em: http://www.ibge.gov.br/cidadesat/topwindow.htm?1. Acesso em: 10 junho 2012 .

GLIESSMAN, S. R. Agroecologia: processos ecológicos em agricultura sustentável. 3. ed. Porto Alegre: UFRGS, 653p

GUZMÁN, E. S. A perspectiva sociológica em Agroecologia: uma sistematização de seus métodos e técnicas. Agroecologia e Desenvolvimento Rural Sustentavél, Porto Alegre, v.3, n.1 p.18-28, jan./mar. 2002. Disponível em: < http://www.projetovidanocampo.com.br/agroecologia/uma_es trategia_de_sustentabilidade_a_partir_da_agroecologia.pdf.

Acesso em: 30/04/2015.

GUZMÁN, E. S. Uma estratégia de sustentabilidade a partir da Agroecologia. Agroecologia e Desenvolvimento Rural Sustentável, Porto Alegre, v.2, n.1, jan./mar.2001.

MOREIRA, R. M.; CARMO, M.S. Agroecologia na construção do Desenvolvimento Rural Sustentável. Agric. São Paulo, São Paulo, v. 51, n. 2, p. 37-56, jul./dez. 2004.

SANT'ANA, A. L. Estratégias dos Agricultores Familiares: Entre a moldagem da intensificação produtiva e a construção de formas diferenciadas de comercialização. Retratos de Assentamentos, v. 14, n. 1, 2011. p. 283-304

SCHMITT. C. J. Transição agroecológica e desenvolvimento rural: um olhar a partir da experiência brasileira. $2^{\circ}$ ed. São Paulo: Expressão popular, 2013. 328.p.

WEZEL, A.; BELLON, S.; DORE, T.; FRANCIS, C.; VALLOD, D.; DAVID, C. Agroecology as a science, a movement and a practice. A review. Agronomy for Sustainable Development, 2009. Disponivel em:< https://www.socla.co/wp-content/uploads/2014/wezelagroecology.pdf $>$. Acesso em: 20 de outubro de 2017.
MARIN, J. O. B. Agricultores familiares e os desafios da transição agroecológica. Revista UFG, nº 7, 2009.

REICHERT, L. J.; GOMES. M. C. Processo de tomada de decisão e a racionalidade administrativa na mudança do sistema de produção convencional para o de base ecológica na agricultura familiar. Agricultura familiar: pesquisa, formação e desenvolvimento. Universidade Federal do Pará, Núcleo de Ciências Agrárias e Desenvolvimento Rural NEAF, v.1, n.9., 2009. pp.121-144.

REICHERT, L. J., MACHADO, J. A. D.; PADILHA, M. C. GOMES. M. C. Compreendendo a tomada de decisão de produtores de batata em transição agroecológica no município de São Lourenço do Sul/RS. In: Congresso De Agroecologia E Agricultura Ecológica Em Galícia, 3, Vigo: Gieea.Uvigo, Espanha. 2009.

REICHERT, L. J.; GOMES, M.C. O processo administrativo e a tomada de decisão de agricultores familiares em transição agroecológica. Revista de la Facultad de Agronomía, La Plata (2013) Vol 112 (2): 105-113. 